\title{
John Cantwell
}

\section{THE LAWS OF NON-BIVALENT PROBABILITY}

\begin{abstract}
Non-bivalent languages (languages containing sentences that can be true, false or neither) are given a probabilitistic interpretation in terms of betting quotients. Necessary and sufficient conditions for avoiding Dutch books - the laws of non-bivalent probability - in such a setting are provided.
\end{abstract}

Consider a language $L$ that contains sentences that under some interpretations are neither true nor false. ${ }^{1}$ Say that $L$, amongst other things, is closed under the two unary connectives $\sim$ ('not') and $\operatorname{Tr}$ ('it is true that') (so that if $A$ is a sentence of $L$, then so are $\sim A$ and $\operatorname{Tr}(A))$ and the binary connective $\vee$ ('or') (so if $A$ and $B$ are sentences of $L$, then so is $A \vee B$ ), with the following truth tables ('-' means that the sentence lacks a truth value, $*$ means that it doesn't matter for the results below whether the sentence is true, false, or neither):

\begin{tabular}{c||c|c}
$A$ & $\sim A$ & $\operatorname{Tr}(A)$ \\
\hline $\mathrm{T}$ & $\mathrm{F}$ & $\mathrm{T}$ \\
$\mathrm{F}$ & $\mathrm{T}$ & $\mathrm{F}$ \\
- & - & $\mathrm{F}$
\end{tabular}

\begin{tabular}{c|ccc}
$A \vee B$ & $\mathrm{~T}$ & $\mathrm{~F}$ & - \\
\hline $\mathrm{T}$ & $\mathrm{T}$ & $\mathrm{T}$ & $\mathrm{T}$ \\
$\mathrm{F}$ & $\mathrm{T}$ & $\mathrm{F}$ & $*$ \\
- & $\mathrm{T}$ & $*$ & $*$
\end{tabular}

\footnotetext{
${ }^{1}$ Examples of linguistic phenomena that have been thought to give rise to non-bivalence include: non-referring singular terms (Strawson (1950), van Frassen (1966)), vagueness (Fine (1975), Kamp (1975)), and indicative conditionals (McGee (1989), McDermott (1996)). The claim that these phenomena introduce non-bivalence is, of course, highly controversial.
} 
With the notions $\sim \operatorname{Tr}$ and $\vee$ in place we can define $T V(A)$ (' $A$ has a truth-value') as $\operatorname{Tr}(A) \vee \operatorname{Tr}(\sim A)$.

Let $\mathcal{I}$ be the class of possible assignments of truth values to the sentences of $L$. A sentence $A$ is truth-determinate if it is either true or false in every assignment. $A$ and $B$ are logically equivalent if every assignment that makes $A$ true (false) makes $B$ true (false) and vice versa. $A$ and $B$ are mutually incompatible if there is no assignment where both $A$ and $B$ are true.

The following is a complete axiomatisation of the notion of probability for this language $L$, which is a function $\operatorname{Pr}$ with domain $L$ such that:

For any truth-determinate sentences $A$ and $B$ :

1. $0 \leq \operatorname{Pr}(A) \leq 1$.

2. If $A$ and $B$ are logically equivalent, then $\operatorname{Pr}(A)=\operatorname{Pr}(B)$.

3. $\operatorname{Pr}(\sim A)=1-\operatorname{Pr}(A)$.

4. $\operatorname{Pr}(A \vee B)=\operatorname{Pr}(A)+\operatorname{Pr}(B)$, if $A$ and $B$ are mutually incompatible.

For every sentence $A$ :

5. $\operatorname{Pr}(A)=\operatorname{Pr}(\operatorname{Tr}(A)) / \operatorname{Pr}(T V(A))$, if $\operatorname{Pr}(T V(A))>0$.

The first four are the standard laws of probability, restricted to truthdeterminate sentences only. The fifth is the law of non-bivalent probability; in a more relaxed vernacular it reads 'the probability of $A$ is the probability that $A$ is true, given that $A$ has a truth value.' ${ }^{2}$ Together they will be called the laws of non-bivalent probability. Their soundness and completeness can be established by a Dutch Book argument based on the betting interpretation of probability.

A (betting) book $\mathcal{B}$ consists of two real-valued functions $Q$ and $S . Q(A)$ is the betting quotient for the sentence $A$ and $S(A)$ is the stake for the bet on $A . S$ is restricted so that it can assign a non-zero stake to only a finite number of sentences.

Books work as follows. The subject whose degrees of belief are to be measured sets the betting quotients $Q$ while the bookie sets the stakes $S$. When the stake $S(A)$ is positive, this amounts to a bet on A (from the subject's perspective): the subject wins money if $A$ is true and looses if $A$ is

\footnotetext{
${ }^{2}$ McDermott (1996) comes close to stating the law of non-bivalent probability when he holds (p.4) that the "degree of assertability" of $A$ is given by the probability $\operatorname{Pr}(A$ is true $\mid A$ has truth value). For reasons that are not made entirely clear, McDermott does not consider degrees of assertability, thus defined, to be subjective probabilities.
} 
false. When $S(A)$ is negative, this amounts to a bet against $A$ : the subject looses money if $A$ is true and wins money if $A$ is false. If $A$ is neither true nor false, any bets on or against $A$ will be canceled. That is, a bet on a sentence $A$ that may turn out to lack truth value is a conditional bet. ${ }^{3}$

The net result of a book $\mathcal{B}$ with regard to an assignment $I$ is:

$$
V(\mathcal{B}, I)=\sum_{I(A)=T}((1-Q(A)) \times S(A))-\sum_{I(A)=F}(Q(A) \times S(A))
$$

For instance, if you have set the betting quotient .75 to $A$ and the bettor has assigned the stake $\$ 10$, then you will win $\$ 2.50$ if $I(A)=T$ and loose $\$ 7.50$ if $I(A)=F$. If the bettor has instead assigned the negative stake $\$-5$, then you will loose $\$ 1.25$ if $I(A)=T$ and you will win $\$ 3.75$ if $I(A)=F$. So if you have set the betting quotient for $A$ to the same value as your subjective probability that $A$, then whatever stake the bettor places on $A$ (be it negative or positive), the resulting bet will for you have an expected monetary outcome of $\$ 0$.

A Dutch book is a betting book $\mathcal{B}$ such that for every interpretation $I$, $V(\mathcal{B}, I)<0$, that is, if a Dutch book can be made against an assignment of betting quotients $Q$, this guarantees that the subject will loose whatever happens. The betting quotients $Q$ can be Dutch booked if the bettor can set the stakes in such a way that the resulting book is a Dutch book. ${ }^{4}$

Theorem 1. Any set of betting quotients that violate the laws of nonbivalent probability can be Dutch booked.

Theorem 2. No set of betting quotients that satisfies the laws of nonbivalent probability can be Dutch booked.

Theorem 3. If Pr is a standard bivalent probability measure (i.e. satisfies laws 1-4) on the truth determinate fragment of the language $L$, then there exists a non-bivalent probability measure $\mathrm{Pr}^{\prime}$ on the full language $L$ such that $\operatorname{Pr}^{\prime}(A)=\operatorname{Pr}(A)$ for every truth determinate sentence $A$ (i.e. $\operatorname{Pr}^{\prime}$ extends $\operatorname{Pr}$ ), furthermore, if two measures $\mathrm{Pr}^{\prime}$ and $\mathrm{Pr}^{\prime \prime}$ extend $\mathrm{Pr}$ in this fashion, then for every $A$ such that $\operatorname{Pr}(T V(A))>0, \operatorname{Pr}^{\prime}(A)=\operatorname{Pr}^{\prime \prime}(A) .^{5}$

\footnotetext{
${ }^{3}$ De Finetti (1937) used conditional bets to give an interpretation of conditional probability, see also Milne (1997).

${ }^{4}$ De Finetti (1937) and Ramsey (1931) used the idea of a Dutch book as a means of justifying the standard laws of probability. They, however, were working with bivalent languages.

${ }^{5}$ Note that the laws say nothing about $\operatorname{Pr}(A)$ when $A$ is not truth determinate and
} 
Non-bivalent probability carries with it a kind of 'probability logic' (for pairs of sentences $A$ and $B$ ). Define:

$A \models B$ if and only if either (i) $A$ and $B$ are logically equivalent, or (ii) $B$ is true in every assignment where $A$ is not false.

Theorem 4. $A \models B$ if and only if for every Pr satisfying laws (1-5): $\operatorname{Pr}(A) \leq$ $\operatorname{Pr}(B)$.

It is mildly ironic that the original Dutch book argument was designed to establish the coherence and completeness of the standard laws of probability, but here it can be used to establish the incoherence of one of the laws in its unrestricted form. Consider the non truth-determinate sentences $A$ and $B$ and say that the probabilities are distributed among the different logical possibilities as follows:

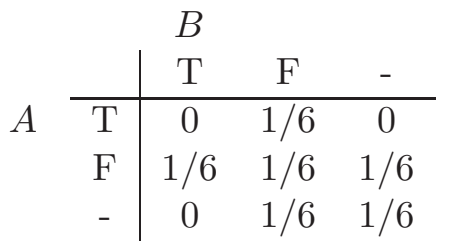

Note that $\operatorname{Pr}(B)=\operatorname{Pr}(A)=\operatorname{Pr}(\operatorname{Tr}(A)) / \operatorname{Pr}(T V(A))=\frac{1 / 6}{2 / 3}=1 / 4$. So $\operatorname{Pr}(A)+\operatorname{Pr}(B)=1 / 2$. The value of $\operatorname{Pr}(A \vee B)$ will vary depending on how we select the truth conditions for disjunction (the values for $*$ in the truth table). If we adopt the plausible assumption that they are symmetric (so that $A \vee B$ is equivalent to $B \vee A$ ) there are three possibilities. (i) The disjunction $A \vee B$ lacks truth value if one of the disjuncts is false and the other lacks truth value. In this case $\operatorname{Pr}(A \vee B)=\operatorname{Pr}(\operatorname{Tr}(A \vee B)) / \operatorname{Pr}(T V(A \vee B))=\frac{1 / 3}{1 / 2}=2 / 3$ so additivity (law 4 above) does not hold. (ii) If $A \vee B$ is instead false as soon as neither disjunct is true we have $\operatorname{Pr}(A \vee B)=1 / 3$, still contradicting law 4. (iii) If, instead, $A \vee B$ is false if one disjunct is false and the other lacks truth value, while $A \vee B$ lacks truth value when both disjuncts lacks truth value, then $\operatorname{Pr}(A \vee B)=6 / 15$ and law 4 is still not valid.

The remaining standard laws, however, are valid also in unrestricted form as long as we are dealing with sentences $A$ such that $\operatorname{Pr}(T V(A)) \neq 0$. For $\operatorname{TV}(A)$ is defined as the truth-determinate $\operatorname{Tr}(A) \vee \operatorname{Tr}(\sim A)$ so, by

$\operatorname{Pr}(T V(A))=0$. A generalisation to a non-bivalent 'Popper-style'-measure (whereby the conditional probability $\operatorname{Pr}(A \mid B)$ is defined even when $\operatorname{Pr}(B)=0$ ) could remedy this, but such a measure does not seem to have any interpretation in terms of betting quotients. 
law 4 (which still holds for truth-determinate sentences), $0<\operatorname{Pr}(\operatorname{Tr}(A)) /$ $\operatorname{Pr}(T V(A)) \leq 1$ and so $0<\operatorname{Pr}(A) \leq 1$ (law 1). Furthermore, if $A$ and $B$ are logically equivalent, then $\operatorname{Tr}(A)$ and $\operatorname{Tr}(B)$ are logically equivalent and $T V(A)$ and $T V(B)$ are logically equivalent. But then $\operatorname{Pr}(\operatorname{Tr}(A)) /$ $\operatorname{Pr}(T V(A))=\operatorname{Pr}(\operatorname{Tr}(B)) / \operatorname{Pr}(T V(A))$ and so $\operatorname{Pr}(A)=\operatorname{Pr}(B)$ (law 2).

Finally, note that $\operatorname{Tr}(A) \vee \operatorname{Tr}(\sim A) \vee \sim T V(A)$ is always true so $\operatorname{Pr}(\operatorname{Tr}(A)$ $\vee \operatorname{Tr}(\sim A) \vee \sim T V(A))=\operatorname{Pr}(\operatorname{Tr}(A))+\operatorname{Pr}(\operatorname{Tr}(\sim A))+\operatorname{Pr}(\sim T V(A))=1$. So $\operatorname{Pr}(\operatorname{Tr}(\sim A))=1-(\operatorname{Pr}(\operatorname{Tr}(A))+\operatorname{Pr}(\sim T V(A)))$ (note also that $\operatorname{Pr}(T V(\sim A))=\operatorname{Pr}(T V(A)))$. Thus (law 3$)$

$$
\begin{aligned}
\operatorname{Pr}(\sim A) & =\operatorname{Pr}(\operatorname{Tr}(\sim A)) / \operatorname{Pr}(T V(\sim A))=\frac{1-(\operatorname{Pr}(\operatorname{Tr}(A))+\operatorname{Pr}(\sim T V(A)))}{\operatorname{Pr}(T V(A))} \\
& =\frac{1-(\operatorname{Pr}(\operatorname{Tr}(A))+1-\operatorname{Pr}(T V(A)))}{\operatorname{Pr}(T V(A))}=\frac{\operatorname{Pr}(T V(A))-\operatorname{Pr}(\operatorname{Tr}(A))}{\operatorname{Pr}(T V(A))} \\
& =1-\frac{\operatorname{Pr}(\operatorname{Tr}(A))}{\operatorname{Pr}(T V(A))}=1-\operatorname{Pr}(A)
\end{aligned}
$$

David Lewis, voicing a complaint against the idea that the standard laws of probability be modified to circumvent his "impossibility theorem" for conditionals, writes:

But if it be granted that "probabilities" of conditionals do not obey the standard laws, I do not see what is to be gained by insisting on calling them "probabilities". (1976, p. 304)

I think Lewis here ties the concept of probability too closely to a particular set of laws. If betting dispositions provide a reasonable model for degrees of belief, it is reasonable to call the result a measure of "subjective probability". If the underlying model does not validate the standard laws of probabilityand it will not if the language contains sentences that can lack truth valueone should have serious qualms about letting the standard laws of probability override the model. The laws of probability evolved in a bivalent context and we should not presuppose that they will remain intact when the context is not bivalent.

\section{Proof of theorems}

Proof of Theorem 1: Note that as long as $A$ and $B$ are truth-determinate, we know that violation of any of the laws 1-4 can be Dutch Booked. Thus we only need to establish that a violation of law 5 can be Dutch booked. 
Assume that $Q(T V(A)) \neq 0$ (as $T V(A)$ is a truth-determinate sentence this means that $0<Q(T V(A)) \leq 1$.

Case 1. Assume that $Q(A)<Q(\operatorname{Tr}(A)) / Q(T V(A)$. Set $S(A)=-1$, $S(\operatorname{Tr}(A))=1$ and $S(T V(A))=-Q(A)$. The payoffs are as follows:

\begin{tabular}{c||c|c|c}
$A$ & $A$ & $\operatorname{Tr}(A)$ & $T V(A)$ \\
\hline $\mathrm{T}$ & $(1-Q(A))(-1)$ & $1-Q(\operatorname{Tr}(A))$ & $(1-Q(T V(A)))(-Q(A))$ \\
$\mathrm{F}$ & $-Q(A)(-1)$ & $-Q(\operatorname{Tr}(A))$ & $(1-Q(T V(A)))(-Q(A))$ \\
- & 0 & $-Q(\operatorname{Tr}(A))$ & $-Q(T V(A))(-Q(A))$
\end{tabular}

Note that the sum of the each row is equal to $Q(A) Q(T V(A))-Q(\operatorname{Tr}(A))$ and, as $Q(A)<Q(\operatorname{Tr}(A)) / Q(T V(A)$, this is less than 0 .

Case 2. Assume that $Q(A)>Q(\operatorname{Tr}(A)) / Q(T V(A))$. Set $S(A)=1$, $S(\operatorname{Tr}(A))=-1$ and $S(T V(A))=Q(A)$.

\begin{tabular}{c||c|c|c}
$A$ & $A$ & $\operatorname{Tr}(A)$ & $T V(A)$ \\
\hline $\mathrm{T}$ & $1-Q(A)$ & $(1-Q(\operatorname{Tr}(A))(-1)$ & $(1-Q(T V(A))) Q(A)$ \\
$\mathrm{F}$ & $-Q(A)$ & $-Q(\operatorname{Tr}(A))(-1)$ & $(1-Q(T V(A))) Q(A)$ \\
- & 0 & $-Q(\operatorname{Tr}(A))(-1)$ & $-Q(T V(A)) Q(A)$
\end{tabular}

Note that the sum of each row is $Q(\operatorname{Tr}(A))-Q(A) Q(T V(A))$ and, as $Q(A)>$ $Q(\operatorname{Tr}(A)) / Q(\operatorname{TV}(A))$, this will always be less than 0 .

Proof of Theorem 2: Assume that the betting quotients satisfy the laws of non-bivalent probability. The proof proceeds by establishing that if there is a Dutch book on sentences containing non-truth-determinate sentences, then a Dutch book on can be made on truth-determinate sentences only, and this cannot happen as these satisfy the standard laws of probability.

So assume that there is an assignment of stakes $S$ such that for every assignment $I, V(S, I)<0$. By assumption there must be some nontruth-determinate sentence $A$ such that $S(A) \neq 0$ (as otherwise only truthdeterminate sentences would be involved in the Dutch Book). Two cases: (1) $Q(A)>0$, and (2) $Q(A)=0$.

Consider the first case. Let $x=Q(A), y=Q(\operatorname{Tr}(A))$ and $z=Q(\sim$ $T V(A))$. Let $S^{\prime}$ be an assignment of stakes that is just like $S$ except that $S^{\prime}(A)=0, S^{\prime}(\operatorname{Tr}(A))=S(A)+S(\operatorname{Tr}(A))$ and $S^{\prime}(\sim T V(A))=x \times S(A)+S(\sim$ $T V(A))$. One can now show that for every assignment $I: V(S, I)=V\left(S^{\prime}, I\right)$. As $S$ and $S^{\prime}$ differ only on the values assigned to $A, \operatorname{Tr}(A)$ and $\sim T V(A)$ we can concentrate on them. 


\begin{tabular}{c||c|c|c}
\multicolumn{4}{c}{$S$} \\
\hline$A$ & $\operatorname{Tr}(A)$ & $\sim T V(A)$ & $A$ \\
\hline $\mathrm{T}$ & $(1-y) S(\operatorname{Tr}(A))$ & $-z S(\sim T V(A))$ & $(1-x) S(A)$ \\
$\mathrm{F}$ & $-y S(\operatorname{Tr}(A))$ & $-z S(\sim T V(A))$ & $-x S(A)$ \\
- & $-y S(\operatorname{Tr}(A))$ & $(1-z) S(\sim T V(A))$ & 0
\end{tabular}

\begin{tabular}{c||c|c|c}
\multicolumn{4}{c}{$S^{\prime}$} \\
\hline$A$ & $\operatorname{Tr}(A)$ & $\sim T V(A)$ & $A$ \\
\hline $\mathrm{T}$ & $(1-y)(S(A)+S(\operatorname{Tr}(A)))$ & $-z(x S(A)+S(\sim T V(A)))$ & 0 \\
$\mathrm{~F}$ & $-y(S(A)+S(\operatorname{Tr}(A)))$ & $-z(x S(A)+S(\sim T V(A)))$ & 0 \\
- & $-y(S(A)+S(\operatorname{Tr}(A)))$ & $(1-z)(x S(A)+S(\sim T V(A)))$ & 0
\end{tabular}

Note first that $x=y /(1-z)($ as $Q(A)=Q(\operatorname{Tr}(A)) / Q(T V(A)))$, i.e. that $x-z x=y, x=y+z x$ and $-x=-y-z x$. Note that $(1-y) S(A)-z x S(A)=$ $S(A)-y S(A)-z x S(A)=S(A)(1-y-z x)=S(A)(1-x)$. From this it follows that $S$ and $S^{\prime}$ give the same payoffs when $I(A)=T$. Next (for $I(A)=F$ ) note that $-y S(A)-z x S(A)=S(A)(-y-z x)=S(A)(-x)$. Finally, (for $I(A)=-)$ note that $-y S(A)+(1-z) S(A) x=-y S(A)+(x-z x) S(A)=$ $-y S(A)+y S(A)=0$.

Now to the second case $(Q(T V(A))=0)$. Note that in this case $Q(\operatorname{Tr}(A))=0$. Let $x=S(A), y=S(\operatorname{Tr}(A))$ and $z=S(\sim T V(A))$. The strategy is the same: to find an assignment of stakes $S^{\prime}$ that (i) coincides with $S$ on every sentence except $A, \operatorname{Tr}(A)$ and $T V(A)$, that (ii) contrary to $S$, assigns stake 0 to $A$, and (iii) the payoff from $S^{\prime}$ is the same as the payoff from $S$ for every assignment $I$. Let $S^{\prime}(A)=0, S^{\prime}(T V(A))=z-Q(A) x$ and $S^{\prime}(\operatorname{Tr}(A))=x+y$. The payoffs for $S$ and $S^{\prime}$ are:

\begin{tabular}{c||c|c|c}
\multicolumn{4}{c}{$S$} \\
\hline$A$ & $T r(A)$ & $\sim T V(A)$ & $A$ \\
\hline $\mathrm{T}$ & $y$ & $z$ & $(1-Q(A)) x$ \\
$\mathrm{~F}$ & 0 & $z$ & $-Q(A) x$ \\
- & 0 & 0 & 0
\end{tabular}

\begin{tabular}{c||c|c|c}
\multicolumn{4}{c}{$S^{\prime}$} \\
\hline$A$ & $\operatorname{Tr}(A)$ & $\sim T V(A)$ & $A$ \\
\hline $\mathrm{T}$ & $x+y$ & $z-Q(A) x$ & 0 \\
$\mathrm{~F}$ & 0 & $z-Q(A) x$ & 0 \\
- & 0 & 0 & 0
\end{tabular}

Note that if $A$ is true then the payoff for $S$ is $y+z+x-Q(A) x$ which is the same as the payoff for $S^{\prime}$. Similarly if $A$ is false the payoff for $S$ is $z-Q(A) x$ which is the same as the payoff for $S^{\prime}$. When $A$ lacks truth value the payoffs are both 0 .

So in both the above cases $S^{\prime}$ contains one non-zero assignment less to non-truth-determinate sentences than $S$. Thus, by iterating the above 
procedure any setting of stakes $S$ that assigns non-zero stakes to a finite number of sentences, is equivalent to a setting of stakes $S^{\prime}$ that assigns nonzero stakes to truth-determinate sentences only.

Proof of Theorem 3: The result is more or less trivial given law 5. Let Pr be the bivalent probability measure. Define:

$$
\operatorname{Pr}^{\prime}(A)= \begin{cases}\operatorname{Pr}(\operatorname{Tr}(A)) / \operatorname{Pr}(T V(A)), & \text { if } \operatorname{Pr}(T V(A))>0, \\ 0, & \text { otherwise. }\end{cases}
$$

Clearly $\operatorname{Pr}^{\prime}$, thus defined, is a non-bivalent probability measure that extends $\operatorname{Pr}$ (note that when $A$ is truth determinate $\operatorname{Pr}(\operatorname{Tr}(A))=\operatorname{Pr}(A)$ and $\operatorname{Pr}(T V(A))=1$ ), furthermore, due to law 5 , any other extension $\operatorname{Pr}^{\prime \prime}$ must coincide with $\operatorname{Pr}^{\prime}$ on every sentence $A$ such that $\operatorname{Pr}(T V(A))>0$.

Proof of Theorem 4: Right-to-left. If $A$ and $B$ are logically equivalent then by law $2 \operatorname{Pr}(A)=\operatorname{Pr}(B)$. So assume that $B$ is true in every assignment where $A$ is not false. Take any probability measure Pr. From the assumption, $\operatorname{Pr}(\neg F(A)) \leq \operatorname{Pr}(\operatorname{Tr}(B))$ (where $F(A)$ is defined: $\operatorname{TV}(A) \wedge \sim \operatorname{Tr}(A)$ ). Two cases. (i) $\operatorname{Pr}(T V(A))=0$. In this case $\operatorname{Pr}(\neg F(A))=1$ and so $\operatorname{Pr}(\operatorname{Tr}(B))=1$ and $\operatorname{Pr}(B)=1$. Thus $\operatorname{Pr}(A) \leq \operatorname{Pr}(B)$ (I am assuming here that a general constraint on probability measures is that $\operatorname{Pr}(X) \leq 1$-this does not follow (when $\operatorname{Pr}(T V(X))=0$ ) from the laws of probability but must be seen as an extra purely conventional postulate). (ii) $\operatorname{Pr}(T V(A))>0$. By law 5, $\operatorname{Pr}(A)=\operatorname{Pr}(\operatorname{Tr}(A)) / \operatorname{Pr}(T V(A))$. As $T V(A)$ is truth determinate we have, by law $4, \operatorname{Pr}(A)=\operatorname{Pr}(\operatorname{Tr}(A)) /(\operatorname{Pr}(\operatorname{Tr}(A))+\operatorname{Pr}(F(A)))$. As $\operatorname{Pr}(\operatorname{Tr}(A)) \leq$ $\operatorname{Pr}(\operatorname{Tr}(B)), \operatorname{Pr}(\operatorname{Tr}(A)) /(\operatorname{Pr}(\operatorname{Tr}(A))+\operatorname{Pr}(F(A))) \leq \operatorname{Pr}(\operatorname{Tr}(B)) /(\operatorname{Pr}(\operatorname{Tr}(B))+$ $\operatorname{Pr}(F(A)))$ and as $\operatorname{Pr}(F(B)) \leq \operatorname{Pr}(F(A)), \operatorname{Pr}(\operatorname{Tr}(B)) /(\operatorname{Pr}(\operatorname{Tr}(B))+$ $\operatorname{Pr}(F(B)))=\operatorname{Pr}(B)$ (by law 5). So $\operatorname{Pr}(A) \leq \operatorname{Pr}(B)$.

Left-to-right. Assume that $\operatorname{Pr}(A) \leq \operatorname{Pr}(B)$ for all Pr satisfying laws 1-5. Assume for reductio that there is some assignment $I$ where $A$ is non-false and $B$ is false. Define, for any truth-determinate $C$ :

$$
\operatorname{Pr}^{*}(C)= \begin{cases}1, & \text { if } C \text { is true at } I \\ 0, & \text { otherwise. }\end{cases}
$$

Pr* can be extended to a full probability measure $\operatorname{Pr}$ by (for any $C$ ):

$$
\operatorname{Pr}(C)= \begin{cases}\operatorname{Pr}^{*}(T r(C)) / \operatorname{Pr}^{*}(T V(C)), & \text { if } \operatorname{Pr}^{*}(T V(C))>0, \\ 1, & \text { otherwise. }\end{cases}
$$


We know that $\operatorname{Pr}(B)=0\left(\right.$ as $\left.\operatorname{Pr}^{*}(F(B))=1\right)$. Now, either $A$ is true at $I$, in which case $\operatorname{Pr}^{*}(\operatorname{Tr}(A))=1$ and so $\operatorname{Pr}(A)=1$. Or $A$ lacks truth value at $I$, in which case $\operatorname{Pr}^{*}(T V(A))=0$ and so $\operatorname{Pr}(A)=1$. In either case: $\operatorname{Pr}(B)<\operatorname{Pr}(A)$ which contradicts our initial assumption.

\section{References}

[De Finetti, 1937] De Finetti, B., "La prévision: Ses lois logiques, ses sources subjectives", Annales de l'Institut Henri Poincaré 7 (1937), 1-68.

[Fine, 1975] Fine, K., "Vagueness, truth and logic", Synthese 30 (1975), 265-300.

[Kamp, 1975] Kamp, H., "Two theories about adjectives", in: E. Keenan, (ed.), Formal Semantics of Natural Language, Cambridge University Press, Cambridge, 1975, pages 123-155.

[Lewis, 1976] Lewis, D., "Probabilities of conditionals and conditional probabilities", Philosophical Review 85 (1976), 297-315.

[McDermott, 1996] McDermott, M., "On the truth conditions of certain 'if'sentences", Philosophical Review 105 (1996), 1-37.

[McGee, 1989] McGee, V., "Conditional probabilities and compounds of conditionals", Philosophical Review 98 (1989), 485-542.

[Milne, 1997] Milne, P., "Bruno de Finetti and the logic of conditional events", British Journal for the Philosophy of Science 48 no. 2 (1997), 195-232.

[Ramsey, 1931] Ramsey, F.P., Truth and probability, Routledge and Kegan Paul, New York, 1931.

[Strawson, 1950] Strawson, P., "On referring", Mind, 59(235) (1950), 320-344.

[van Frassen, 1966] van Frassen, B. C., "Singular terms, truth value gaps, and free logic", Journal of Philosophy, 63(17) (1966) 481-495.

John CANTwELL

Division of Philosophy

Royal Institute of Technology

Stockholm, Sweden

cantwell@infra.kth.se 